\title{
Construction of a Salmonella Gallinarum ghost as a novel inactivated vaccine candidate and its protective efficacy against fowl typhoid in chickens
}

Atul A Chaudhari, Chetan V Jawale, Sam Woong Kim and John Hwa Lee*

\begin{abstract}
In order to develop a novel, safe and immunogenic fowl typhoid (FT) vaccine candidate, a Salmonella Gallinarum ghost with controlled expression of the bacteriophage PhiX174 lysis gene E was constructed using pMMP99 plasmid in this study. The formation of the Salmonella Gallinarum ghost with tunnel formation and loss of cytoplasmic contents was observed by scanning electron microscopy and transmission electron microscopy. No viable cells were detectable $24 \mathrm{~h}$ after the induction of gene $E$ expression by an increase in temperature from $37^{\circ} \mathrm{C}$ to $42{ }^{\circ} \mathrm{C}$. The safety and protective efficacy of the Salmonella Gallinarum ghost vaccine was tested in chickens that were divided into four groups: group A (non-immunized control), group B (orally immunized), group C (subcutaneously immunized) and group D (intramuscularly immunized). The birds were immunized at day 7 of age. None of the immunized animals showed any adverse reactions such as abnormal behavior, mortality, or signs of FT such as anorexia, depression, or diarrhea. These birds were subsequently challenged with a virulent Salmonella Gallinarum strain at 3 weeks post-immunization (wpi). Significant protection against the virulent challenge was observed in all immunized groups based on mortality and post-mortem lesions compared to the non-immunized control group. In addition, immunization with the Salmonella Gallinarum ghosts induced significantly high systemic IgG response in all immunized groups. Among the groups, orally-vaccinated group B showed significantly higher levels of secreted IgA. A potent antigen-specific lymphocyte activation response along with significantly increased percentages of $\mathrm{CD}^{+}$and $\mathrm{CD} 8^{+} \mathrm{T}$ lymphocytes found in all immunized groups clearly indicate the induction of cellular immune responses. Overall, these findings suggest that the newly constructed Salmonella Gallinarum ghost appears to be a safe, highly immunogenic, and efficient non-living bacterial vaccine candidate that protects against FT.
\end{abstract}

\section{Introduction}

The Gram-negative bacterium, Salmonella enterica serovar Gallinarum (Salmonella Gallinarum), is one of the major intracellular bacterial pathogens that causes fowl typhoid (FT), a septicemic disease of domestic birds, primarily chickens [1,2]. Salmonella Gallinarum infection is specifically limited to avian species and rarely causes food poisoning in humans [2]. FT is manifested by acute mortality, usually $60-70 \%$, and severe inflammation including hepatitis, splenitis, typhlitis, and omphalitis,

\footnotetext{
* Correspondence: johnhlee@jbnu.ac.kr

College of Veterinary Medicine, Chonbuk National University, Jeonju 561-756, Republic of Korea
}

which results in significant economic losses to the poultry industry worldwide. The eradication of this disease is extremely problematic in countries where the ambient temperature necessitates the use of open-sided housing [3-5].

There have been several attempts to prevent FT by vaccination [5-9]. The rough mutant strain Salmonella Gallinarum 9R developed 50 years ago has been examined due to its protective efficacy against FT [9]. However, the use of live Salmonella Gallinarum 9R vaccine is limited to layer breeds older than 6-weeks and is associated with several disadvantages such as insufficient protection, low growth rate [6], and residual virulence 
that can cause hepatitis and splenic lesions in chicks [10]. Other trials with live attenuated Salmonella vaccines have shown successful induction of both mucosal and systemic immunity [11,12]. However, the major drawbacks with using these live vaccines are the safety of the animals and environmental contamination via fecal shedding. The alternative for these live vaccines could be the use of subunit or inactivated vaccines. The administration of a subunit vaccine along with an adjuvant has been shown to offer better protection than an experimental live vaccine [6]. However, common inactivation methods such as the use of formalin or heat for producing conventional inactivated vaccines often result in reduced or altered antigenic characteristics of the vaccines, and could be responsible for inducing impaired immune responses $[13,14]$. Therefore, a vaccine that can be safely administered to chickens (especially at a young age) to obtain desired immune responses and offer sufficient protection from FT is needed.

The bacterial ghost (BG) represents an innovative approach in non-living vaccine technology for producing safe and potent vaccines against a wide variety of infectious diseases [15-18]. Bacterial ghosts are empty cell envelopes that are produced by the controlled expression of the phiX174 lysis gene $E$ in Gram-negative bacteria. Expression of the lysis gene $E$ leads to the formation of trans-membrane tunnels through which the cytoplasm containing the bacterial genome $[15,18]$ and plasmids is expelled due to high osmotic pressure inside the cell. The resulting bacterial ghosts are believed to retain all functional, morphological, and immunogenic determinants on the cell surface, and are able to induce cellular and humoral immune responses, resulting in effective immunoprotection [19]. Following ghost formation, even highly sensitive and fragile structures such as pili are preserved $[20,21]$. Since lysis tunnel formation is restricted to only a small part of the total cell surface and lysis by gene $E$ does not chemically or physically disrupt bacterial surface structures, the resulting ghosts share the functional and antigenic determinants of the envelope with their living counterparts; thus, the ghosts can elicit prolonged and profound immunity [22-25]. In this study we constructed Salmonella Gallinarum ghosts using a plasmid containing the phiX174 lysis gene $E$. These Salmonella Gallinarum ghosts appear to be a safe vaccine candidate that significantly induces cellular and humoral immune responses, and confers significant protection against virulent Salmonella Gallinarum infection in chickens.

\section{Materials and methods}

\section{Ghost plasmid construction and growth conditions}

Bacterial strains and plasmids used in this study are listed in Table 1. Plasmid pHCE GAPDH ghost 37SDM carrying the parent ghost cassette was constructed as previously described [26]. PCR amplification of the ghost cassette was performed using pHCE GAPDH ghost 37SDM as a template and the primers ghost-F-XbaI (5'TCTAGAGACCAGAACACCTTGCCGATC-3') and ghostR-XbaI (5' - TCTAGAACATTACATCACTCCTTCCG-3'). The amplified DNA segment was cloned into a T-easy vector (Promega, Madison, WI, USA), and designated as pMMP99. JOL394, a wild-type Salmonella Gallinarum (Salmonella Gallinarum) strain, was used for the construction of the ghost vaccine strain. The plasmid pMMP99 was used to transform JOL394 cells on LB agar containing $50 \mu \mathrm{g} / \mathrm{mL}$ of ampicillin; the resulting strain was denoted as JOL1277. All strains were preserved in LB broth with $20 \%$ glycerol and stored at $-80{ }^{\circ} \mathrm{C}$ until use. Phosphate buffered saline (PBS, $\mathrm{pH}=7.4$ ) was used to resuspend the Salmonella Gallinarum ghost vaccine as well as the challenge strain.

\section{Production of the salmonella gallinarum ghosts}

JOL1277 cells harboring pMMP99 were inoculated into $20 \mathrm{~mL}$ of $\mathrm{LB}$ broth containing $50 \mu \mathrm{g} / \mathrm{mL}$ of ampicillin. The cultures were incubated at $37^{\circ} \mathrm{C}$ with slow agitation until the optical density (OD) at $600 \mathrm{~nm}$ was $0.3-0.4$. To induce gene $E$-mediated lysis, the temperature was increased to $42{ }^{\circ} \mathrm{C}$. After increasing the temperature from 37 to $42{ }^{\circ} \mathrm{C}$, lysis was monitored by performing viable cell counts at different time points $(6,12,18$ and

Table 1 Bacterial strains and plasmids used in this study

\begin{tabular}{|c|c|c|}
\hline Strains/plasmid & Description & References \\
\hline \multicolumn{3}{|l|}{ E. coli } \\
\hline Top 10 & F-mcrA (mrr-hsdRMS-mcrBC) F80 lacZ M15 lacX74 recA1 ara 139(ara-leu)7697 galU galK rpsL (Str') endA1 nupG & Invitrogen \\
\hline \multicolumn{3}{|c|}{ Salmonella Gallinarum } \\
\hline JOL394 & Salmonella Gallinarum, isolated from chicken & This study \\
\hline JOL 1277 & Salmonella Gallinarum JOL394 containing pMMP99 & This study \\
\hline \multicolumn{3}{|l|}{ Plasmids } \\
\hline T-vector & Cloning vector; pUCori Amp ${ }^{R}$ & Promega \\
\hline pMMP99 & A derivative of T-easy carrying ghost cassette & This study \\
\hline
\end{tabular}


$24 \mathrm{~h})$. After completion of lysis, bacterial ghosts were harvested by centrifugation $(4000 \times g$ for $10 \mathrm{~min})$, washed three times with sterile PBS, suspended in PBS, and stored at $-20{ }^{\circ} \mathrm{C}$.

\section{Scanning electron microscopy (SEM) and transmission electron microscopy (TEM)}

The morphological features of the Salmonella Gallinarum ghosts (JOL1277) and a Salmonella Gallinarum wild-type strain (JOL394) as a control were examined by scanning electron microscopy (SEM) and transmission electron microscopy (TEM) as previously described with minor modifications $[27,28]$. Briefly, cell materials were harvested by centrifugation $(4000 \times g$ for $10 \mathrm{~min})$ and fixed in $2.5 \%$ glutaraldehyde in PBS ( $\mathrm{pH} 7.4$ ). This was followed by post-fixation in $1 \%$ aqueous osmium tetroxide and then serial dehydration in acetone. Samples were critical point dried and coated with a gold-palladium alloy for subsequent observation by SEM (JSM-5200, JEOL, Japan). To prepare negatively-stained TEM specimens, a drop of the Salmonella Gallinarum ghost suspension was placed on carbon-coated copper grids. The grids were allowed to stand for $2 \mathrm{~min}$ to permit films to form on the grids, the extra solution was removed using blotting paper, and the grids were dried. TEM was performed with a HITACHI-JP/H7600 microscope (Hitachi Science Systems, Japan) operated at an accelerating voltage of $100 \mathrm{kV}$.

\section{Evaluating the safety dose of the salmonella gallinarum ghost vaccine candidate in chickens}

All animal experiments described in this study were conducted with approval (CBU 2011-0017) from the Chonbuk National University Animal Ethics Committee in accordance with the guidelines of the Korean Council on Animal Care. One-day old female Brown Nick chickens were used for all the experiments, and provided with water and antibiotic-free feed ad libitum. To determine the safety and appropriate dose for inoculation of a ghost vaccine, a preliminary experiment was carried out in chickens. The chickens divided into three groups ( $n=15$ per group), were inoculated via oral route using $10^{7}, 10^{8}$ and $10^{9}$ bacterial ghosts at day 7 of age. The control group was inoculated orally with PBS. The general condition of the birds was evaluated daily until 3 weeks post-immunization (wpi). Mortality and the development of clinical symptoms of FT such as anorexia, depression, and greenish-tinted diarrhea were monitored. Loss of appetite, feed residue, and low responsiveness to external stimuli were monitored to identify anorexia and depression. The birds were subsequently challenged at 21 days post-immunization with $100 \mu \mathrm{L}$ of a suspension containing $1 \times 10^{6} \mathrm{CFU}$ of a wild-type Salmonella Gallinarum JOL394 strain and assessed for mortality. Before the actual vaccination-challenge experiment, a small experiment was carried out to monitor the adverse effects of the intramuscular and subcutaneous inoculation with Salmonella Gallinarum ghost vaccine in chickens. The birds ( $n=10 /$ route) were inoculated with PBS containing $10^{8}$ Salmonella Gallinarum ghosts via the intramuscular and subcutaneous route. The control birds ( $n=10 /$ route) received only PBS. None of the birds exhibited any adverse inflammatory reactions when inoculated with Salmonella Gallinarum ghost vaccine or PBS by intramuscular and subcutaneous routes.

\section{Salmonella gallinarum ghost vaccine protection against bacterial challenge}

Based on the previous safety dose evaluation data, Salmonella Gallinarum ghosts were inoculated at $10^{10}$ inoculation dose by oral route and $10^{8}$ Salmonella Gallinarum ghosts (100 times less than the oral dose) for intramuscular or subcutaneous inoculation. All groups of chickens were immunized with the bacterial ghosts at the $7^{\text {th }}$ day of age. In group B, the birds were orally inoculated with the Salmonella Gallinarum ghosts at a concentration of $1 \times 10^{10}$ cells $/ 0.1 \mathrm{~mL} /$ chicken. In group $\mathrm{C}$, the chickens subcutaneously received the ghosts in $0.1 \mathrm{~mL}$ of a suspension containing $1 \times 10^{8}$ cells/chicken. Group D birds were injected intramuscularly with $0.1 \mathrm{~mL}$ of the Salmonella Gallinarum ghost suspension containing $1 \times 10^{8}$ cells/chicken. The control group A was kept as non-immunized control. Post immunization, the birds were monitored for mortality and any detrimental effects induced by Salmonella Gallinarum ghost vaccine as described above. The birds in all groups were challenged with $100 \mu \mathrm{L}$ of a suspension containing $1 \times 10^{6} \mathrm{CFU}$ of a wild-type Salmonella Gallinarum JOL394 strain on day 21 post-immunization. The mortality rate was assessed daily for 14 days after the challenge. All birds were then sacrificed and examined for macroscopic lesions. Lesion scores were determined and recorded using a system similar to the one previously described [29-31] with modifications. Each gross hepatic and splenic lesion was assigned a score of $0,1,2$, or 3 . Hepatic and splenic lesion scores were considered moderate or severe depending on organ enlargement and the presence of white necrotic foci. A score of 0 indicated no lesions whereas a score of 1 was assigned to indicate hepatomegaly or splenomegaly. The presence of over 10 white necrotic foci on the liver and spleen was scored as 2 , and the presence of more than 10 white foci along with the liver and spleen enlargement was scored as 3 . The lesion score for dead birds in all groups was 3. A mean lesion score per group was determined, and differences among the groups were statistically analyzed. Upon post-mortem examination, the presence of the challenge strain in internal organs of birds with FT 
lesions was investigated. For bacterial recovery, a sample of the liver and spleen was directly inoculated on BGA and incubated overnight at $37{ }^{\circ} \mathrm{C}$. Thereafter, the organs were minced and suspended in buffered peptone water (Becton, Dickinson and Company), and the homogenate was incubated overnight at $37{ }^{\circ} \mathrm{C}$ with continuous shaking, followed by culture in Rappaport- Vassiliadis R10 broth (Becton, Dickinson and Company) for $48 \mathrm{~h}$ at $42^{\circ}$ C. A loop of the enrichment broth was streaked onto BGA and the Salmonella- type colonies were examined after incubation at $37^{\circ} \mathrm{C}$ for $24 \mathrm{~h}$.

\section{Collection of plasma and samples for measuring intestinal secretory $\lg \mathrm{A}(\mathrm{s} \lg \mathrm{A})$}

Plasma and intestinal washes were collected from five birds in each group to evaluate the immune responses at each week post-immunization. The plasma samples were obtained by centrifugation of the peripheral blood. The intestinal wash samples were collected as previously described with some modifications [32]. Briefly, the birds were orally administered a lavage solution [ $6 \mathrm{mM}$ polyethylene glycol (PEG; 3350 molecular weight), $40 \mathrm{mM}$ $\mathrm{Na}_{2} \mathrm{SO}_{4}, 20 \mathrm{mM} \mathrm{NaHCO}, 10 \mathrm{mM} \mathrm{KCl}$; and $25 \mathrm{mM}$ $\mathrm{NaCl}, \mathrm{pH}=7.6)]$ at $5 \mathrm{~mL} / \mathrm{kg}$ of body weight. The birds were individually placed in clean empty buckets and monitored for $30 \mathrm{~min}$ until they began to excrete feces. At this time, a sterile solution of $5 \%$ pilocarpine (Sigma, St. Louis, MO, USA) in water was injected intramuscularly at $50 \mathrm{mg} / \mathrm{kg}$ body weight. Administration of pilocarpine was followed by immediate and intermittent excretion of intestinal fluid for $30 \mathrm{~min}$. After collection, the intestinal fluid was vortexed and centrifuged at $700 \times g$ for $10 \mathrm{~min}$. One $\mathrm{mL}$ of the supernatant was saved and added to $10 \mu \mathrm{L}$ of a $100 \mathrm{mM}$ phenylmethanesulfonyl fluoride (PMSF; Sigma,) solution, $10 \mu \mathrm{L}$ of $1 \%$ $(\mathrm{w} / \mathrm{v})$ sodium azide, and $10 \mu \mathrm{L}$ of $5 \%(\mathrm{w} / \mathrm{v})$ bovine serum albumin. The samples were stored at $-20{ }^{\circ} \mathrm{C}$ until use.

\section{Humoral immune response evaluation}

An indirect enzyme-linked immunosorbant assay (ELISA) was performed with an outer membrane protein (OMP) extracted from the JOL394 Salmonella Gallinarum as described before [30]. The presence of plasma IgG and intestinal sIgA against the OMP was identified using a chicken IgG and IgA ELISA quantitation kit (Bethyl Laboratories, Montgomery, TX, USA). The wells of a Microlon ${ }^{\circledR}$ ELISA plate (Greiner Bio-One $\mathrm{GmbH}$, Frickenhausen, Germany) were coated with $100 \mu \mathrm{L}$ of OMP at a concentration of $0.2 \mathrm{mg} / \mathrm{mL}$. Wells were incubated with a 1:250 dilution of plasma for $1 \mathrm{~h}$, followed by incubation with a 1:100 000 dilution of horseradish peroxidase (HRP)-conjugated goat anti-chicken IgG (Bethyl Laboratories, Montgomery, TX, USA) for $1 \mathrm{~h}$. The bound HRP activity was measured using $o$ - phenylmethylsulfonyl fluoride (Sigma-Aldrich). The optical density at $492 \mathrm{~nm}$ was measured with an ELISA reader after the reaction was stopped with $50 \mu \mathrm{L}$ of $3 \mathrm{M}$ sulfuric acid. The sIgA concentrations were quantified using procedures similar to the ones that were used to measure plasma IgG levels (except that the intestinal wash samples were diluted 1:100).

\section{Cellular immune response evaluation \\ Antigen-specific cell activation of peripheral blood mononuclear cells}

A lymphocyte activation assay was carried out as previously described to evaluate cell-mediated immunity in the immunized groups [33]. Soluble antigen was prepared from the Salmonella Gallinarum wild-type strain JOL394 as previously described [31]. Briefly, the bacterial cell suspension was sonicated for $2 \mathrm{~min}$, and centrifuged at $5000 \times g$ for $60 \mathrm{~min}$ at $4{ }^{\circ} \mathrm{C}$. Supernatant containing the sonicated bacterial cell protein suspension (sbcp) was used as the soluble antigen. Peripheral blood mononuclear cells (PBMC) were separated from five chickens randomly selected from each group using a gentle swirl technique [34] on day 21 post-immunization. Trypan blue dye exclusion testing was done to determine cell viability. A $100 \mu \mathrm{L}$ suspension of viable mononuclear cells containing $1 \times 10^{5}$ cells/mL added to RPMI-1640 medium supplemented with $10 \%$ fetal calf serum, $2 \mathrm{mM}$ L-glutamine, $50 \mathrm{U} / \mathrm{mL}$ penicillin, $50 \mu \mathrm{g} / \mathrm{mL}$ streptomycin, and $2 \mu \mathrm{g} / \mathrm{mL}$ fungizone. The suspension was incubated in triplicate in the wells of 96-well tissue culture plates with $50 \mu \mathrm{L}$ of medium alone (negative control) or medium containing $4 \mu \mathrm{g} / \mathrm{mL}$ of sbcp at $40{ }^{\circ} \mathrm{C}$ in a humidified $5 \% \mathrm{CO}_{2}$ atmosphere for $72 \mathrm{~h}$. The $10 \mu \mathrm{g} / \mathrm{mL}$ Concanavalin A (ConA, Sigma, St. Louis, MO, USA) stimulated lymphocyte cells were kept as a positive control and activity of the stimulated cell suspension was measured. Activation of stimulated lymphocytes was measured using adenosine triphosphate (ATP) bioluminescence as a cell viability marker with a ViaLight ${ }^{\circledR}$ Plus Kit (Lonza, Rockland, ME, USA), which estimated mitochondrial activity [35]. The emitted light intensity was measured using a TriStarLB941 luminometer (Berthold Technologies GmbH and Co., Bad Wildbad, Germany) with an integrated program of $1 \mathrm{~s}$ duration. The values for each group are expressed as relative luminescence units (RLU).

\section{Flow cytometry analysis of $C D 3^{+}, C D 4^{+}$and $C D 8^{+}$subsets}

$\mathrm{T}$ cell markers such as $\mathrm{CD}^{+}, \mathrm{CD}^{+}$, and $\mathrm{CD}^{+}$were examined by collecting peripheral blood mononuclear cells (PBMC) from all the groups on day 7 postimmunization [36]. The cell suspensions were prepared at a concentration of $1 \times 10^{6}$ cells $/ \mathrm{mL}$ in cold PBS. The cells were washed three times in PBS and then incubated 
with $0.1 \mathrm{~mL}$ of appropriately diluted fluorescein isothiocyanate (FITC)-labeled anti-CD3, biotin (BIOT)-labeled anti-CD4 and phycoerythrin (PE)-labeled anti-CD8a (SouthernBiotech, Birmingham, AL, USA) monoclonal antibodies in the dark at $4{ }^{\circ} \mathrm{C}$ for $30 \mathrm{~min}$. After washing three times with cold PBS, the cells were incubated with $0.1 \mathrm{~mL}$ of appropriately diluted allophycocyanin (APC)labeled streptavidin (SouthernBiotech, Birmingham, USA) monoclonal antibody in the dark at $4{ }^{\circ} \mathrm{C}$ for $30 \mathrm{~min}$. After incubation, all samples were washed three times with cold PBS, resuspended in $0.5 \mathrm{~mL}$ of PBS, and analyzed with a flow cytometer (BD Biosciences, NJ, USA). FACS analysis of 10000 events was performed using CellQuest software (BD Biosciences).

\section{Statistical analysis}

All data are expressed as the mean \pm standard deviation (SD) unless otherwise specified. Analyses were performed with SPSS version 16.0 software (SPSS, Chicago, IL, USA). A non-parametric chi-square test was used to analyze significant differences in mortality and gross lesion scores. A one way ANOVA with post hoc Bonferroni adjustments was used to analyze statistical differences in immune responses between the immunized groups and unimmunized control group. Differences were considered to be statistically significant when the $P$-values were $\leq 0.05$ or 0.01 .

\section{Results}

Construction of the ghost cassette and lysis of salmonella gallinarum bacterial ghosts

The ghost cassette contained the $\mathrm{cI} \mathrm{P}_{\mathrm{R}}$ promoter from bacteriophage lambda and $E$ gene from phiX174 fused at transcriptional and translational levels. A new ghost cassette designated as ghost SDM37 was constructed as described in a previous study [26]. The SDM37 ghost system was prepared by introducing a mutation in an operator region encoding the $R$ gene. The point-mutated site in ghost SDM37 was located at OR2 which consisted of 17 nucleotides (nts). Among the $17 \mathrm{nts}$, the ninth nucleotide, $\mathrm{C}$, was replaced with $\mathrm{T}$ in ghost SDM37. Because of this, the strain carrying the SDM37 ghost system could grow at temperatures lower than $37^{\circ} \mathrm{C}$ but were unable to grow at temperatures higher than $37^{\circ} \mathrm{C}$ due to the induction of lysis.

A plasmid (pMMP99) containing the ghost cassette was used to transform a wild-type Salmonella Gallinarum strain, JOL394. Production of Salmonella Gallinarum ghosts in a $25 \mathrm{~mL}$ liquid culture of randomly selected clones was accomplished by increasing the temperature up to $42{ }^{\circ} \mathrm{C}$ to activate gene $E$-mediated lysis. The OD of the transformed Salmonella Gallinarum culture decreased during $6 \mathrm{~h}$ after induction of gene $E$ expression due to cell lysis and remained constant for the next $24 \mathrm{~h}$ until the bacterial ghosts were harvested (Table 2). Cell viability also decreased after lysis induction until the time of ghost harvesting (Table 2). The concentration of viable bacteria, $6 \mathrm{~h}$ after lysis induction, decreased $10^{5}$-fold orders of magnitude (Table 2). Lysis was completed $24 \mathrm{~h}$ after the initiation at which time no viable cells were detected.

Formation of the Salmonella Gallinarum ghosts was examined by SEM and TEM. Electron microscopic analysis revealed the formation of lysis pores in the Salmonella Gallinarum ghosts (indicated by arrowheads in Figure 1a). Pores resulting from gene $E$ tunnel formation could be seen either in the pole region or central division region (Figure 1a). TEM examination showed that the Salmonella Gallinarum ghost cells were empty due to the loss of cytoplasmic material and had collapsed cell envelopes compared to the wild-type cells (Figure 1b).

\section{Safety of the ghost vaccine candidate in chickens}

Seven-day-old chickens were inoculated with the Salmonella Gallinarum ghost strain. To evaluate the safety of the vaccine, parameters including clinical symptoms and mortality were measured post-immunization. No vaccination associated mortality was observed in any of the immunized groups. Furthermore, the birds remained healthy without any symptoms of anorexia, diarrhea, or depression for 3 weeks after inoculation with the ghost strain.

\section{Humoral immune responses}

Humoral immune responses specific for the OMP antigen extracted from wild-type Salmonella Gallinarum were evaluated by indirect ELISA. Systemic IgG and mucosal sIgA levels were determined at every week postimmunization by measuring specific IgG levels in plasma and IgA concentrations in intestinal wash fluid. The immunized birds in group B (orally immunized), group C (subcutaneously immunized), and group D (intramuscularly immunized) had significantly higher plasma IgG levels than the group A control birds (Figure 2a) every

\begin{tabular}{|c|c|c|}
\hline Incubation hours ${ }^{a}$ & $\mathrm{OD}_{600}$ & $\mathrm{CFU}^{\mathrm{b}}$ \\
\hline 0 & 0.320 & $2.5 \times 10^{9}$ \\
\hline 6 & 0.188 & $1.8 \times 10^{4}$ \\
\hline 12 & 0.178 & $4 \times 10^{3}$ \\
\hline 18 & 0.150 & $1 \times 10^{3}$ \\
\hline 24 & 0.167 & 0 \\
\hline
\end{tabular}

${ }^{a}$ The cultures were collected at different time intervals such as $0,6,12,18$ and $24 \mathrm{~h}$ after the induction of lysis.

${ }^{b}$ The viable cell count was measured as colony forming units (CFU)/mL. 

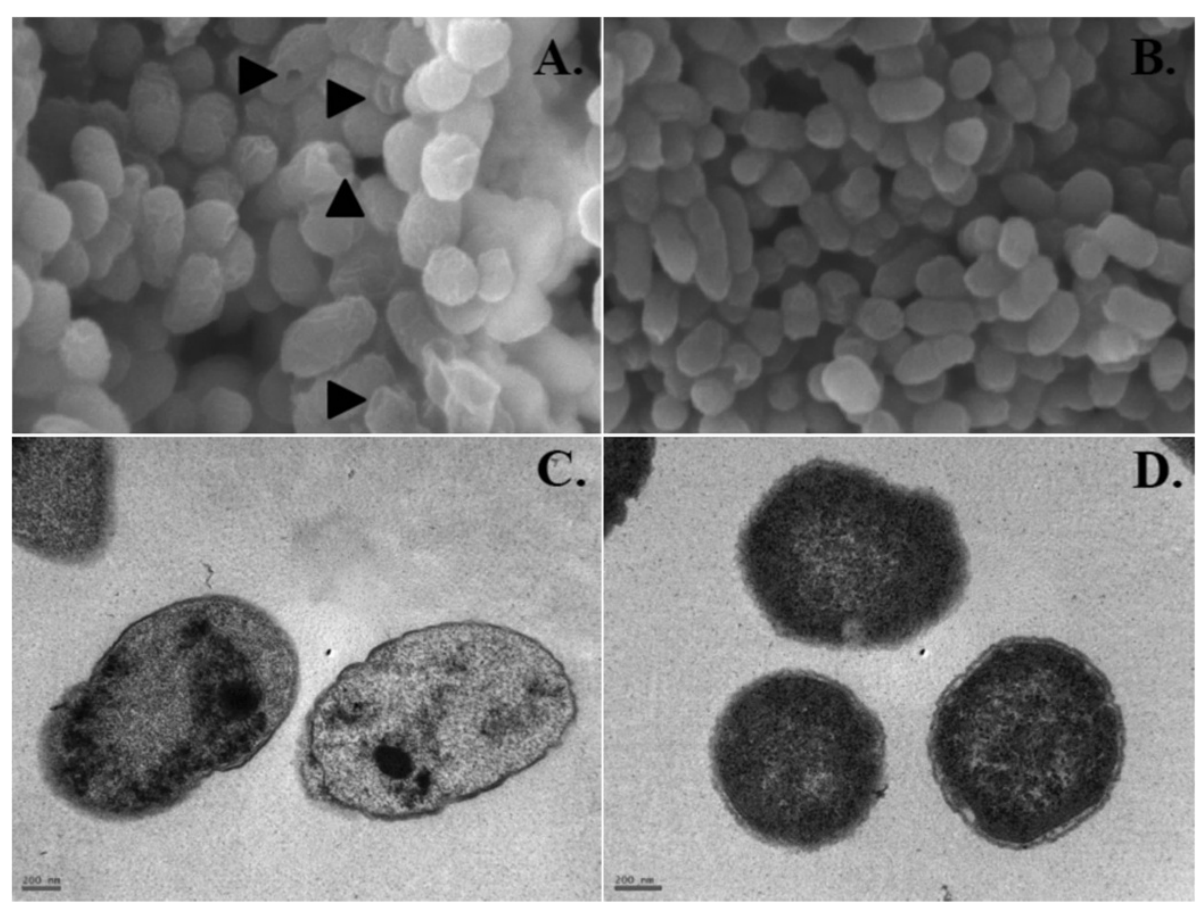

Figure 1 Evaluation of Salmonella Gallinarum ghosts (JOL1277) and Salmonella Gallinarum (JOL394) by SEM (A and B) and TEM (C and D). A) Salmonella Gallinarum ghosts. Arrow shows the trans-membrane lysis tunnel. B) Naive Salmonella Gallinarum examined by SEM. C) Loss of cytoplasmic material from Salmonella Gallinarum ghosts. D) Naive Salmonella Gallinarum examined by TEM.

week post-immunization (wpi). The increases in $\operatorname{IgG}$ levels at 3 wpi in the immunized groups were highly significant compared to the non-immunized group (Figure 2a). Sequential monitoring of intestinal sIgA antibodies showed significantly increased levels in the orally immunized group B compared to the control group (Figure $2 \mathrm{~b}$ ). There was no significant increase in intestinal sIgA levels in groups C or D (Figure 2b).
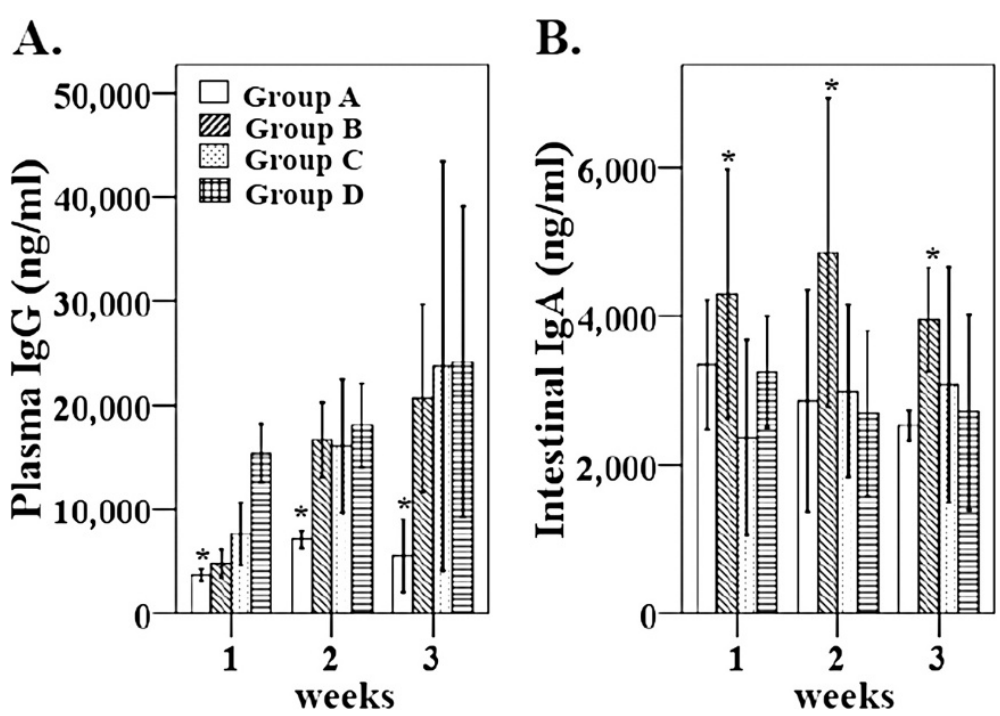

Figure 2 The plasma lgG and intestinal slgA levels in chickens against the outer membrane protein (OMP). A) Plasma lgG levels against specific antigens were determined with the chicken IgG quantitative ELISA. B) The slgA levels in intestinal wash fluids against OMP antigen were quantified using the chicken IgA quantitative ELISA. Antibody levels were expressed as mean \pm S.D. values for each week post-immunization. The values were considered statistically significant, if $p$ values were $\leq 0.05$ ( ${ }^{*} p \leq 0.05$ ). Group A, non-immunized control; group B, orally immunized; group C, immunized subcutaneously; group D, intramuscularly immunized. 


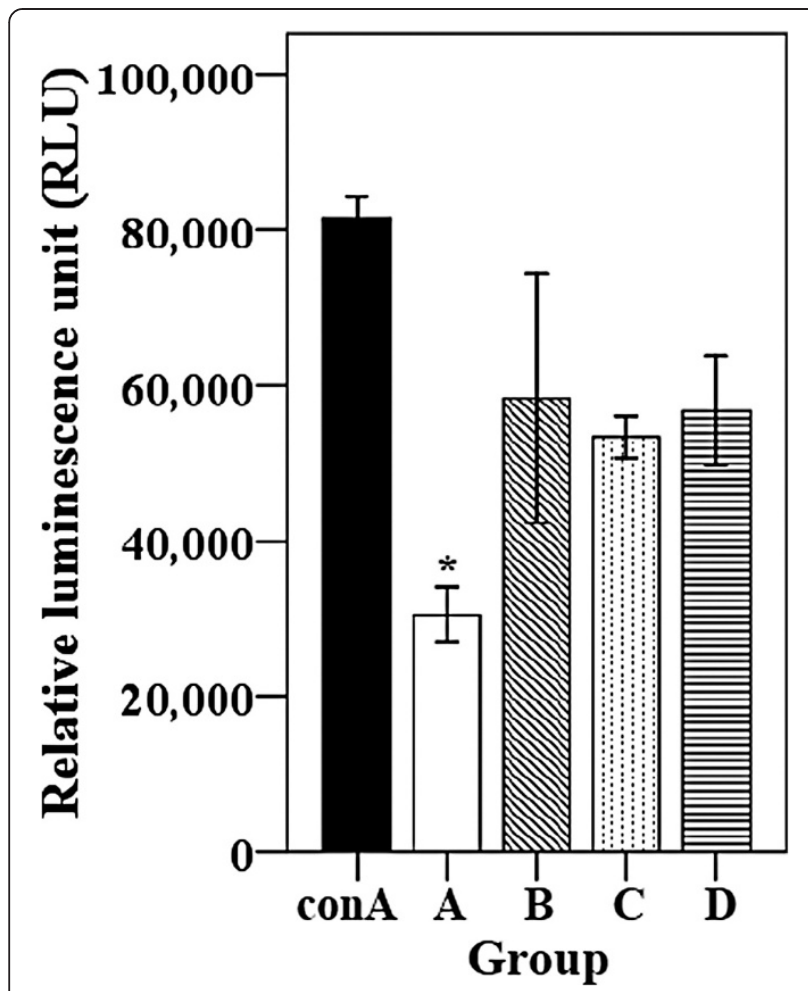

Figure 3 The lymphocyte stimulation responses determined at 3-week-postimmunization against the sbcp Antigen. The stimulation index of lymphocyte sample from the chickens was determined by the peripheral lymphocyte activation assay. The values were considered statistically significant, if $p$ values were $\leq 0.05$ $\left({ }^{*} p \leq 0.05\right)$. Group A, non-immunized control; group B, orally immunized; group $C$, immunized subcutaneously; group $D$, intramuscularly immunized.

\section{Cellular immune responses}

\section{Lymphocyte activation assay}

In order to evaluate the cell-mediated immune responses, lymphocyte activation after stimulation with soluble antigen was measured by analyzing PBMC isolated from immunized and non-immunized birds at 3 wpi. The immunized birds showed significant activation responses compared to control group A (Figure 3). The immunized group RLU values differ significantly compared to those of the control group, suggesting a dramatic increase of lymphocyte activation after antigen stimulation.

\section{Flow cytometry analysis of $\mathrm{CD} 4^{+}$and $\mathrm{CD} 8^{+} \mathrm{T}$ cells}

Changes in $\mathrm{T}$ cell populations after immunization correlated with comparable changes in serum antibody levels. Therefore, we measured the $\mathrm{CD}^{+}{ }^{+}$( $\mathrm{T}$ helper cells) and $\mathrm{CD}^{+}$(cytotoxic $\mathrm{T}$ cells) $\mathrm{T}$ cell subpopulations among PBMC from the immunization groups by flow cytometry. The relative numbers of both $\mathrm{CD} 4^{+}$and $\mathrm{CD} 8^{+}$lymphocytes significantly increased in all the immunized groups (Figure 4). The immunized group had statistically $(p<0.05)$ higher numbers of $\mathrm{CD}^{+} \mathrm{CD}^{+}$, and $\mathrm{CD}^{+}$ $\mathrm{CD}^{+} \mathrm{T}$ cells than the non-immunized control group (Figure $4 \mathrm{a}$ and $\mathrm{b}$ ). On day 7 post-immunization, the numbers of $\mathrm{CD}^{+} \mathrm{CD}^{+}$and $\mathrm{CD}^{+} \mathrm{CD}^{+} \mathrm{T}$ cells among the PBMC populations of all immunized chickens were approximately 1.5 - and 2.5-fold greater than the nonimmunized control group, respectively (Figure 4).

\section{Protective efficacy of bacterial ghosts against virulent challenge in chickens}

In the preliminary experiment for determination of safety dose for inoculation, it was observed that the birds immunized with $10^{7}, 10^{8}$ and $10^{9}$ showed 46.6, 33.5 and $26 \%$ mortality against the virulent challenge, respectively (Table 3). Based on this, the birds were immunized with $10^{10}$ and $10^{8}$ bacterial ghosts via oral and intramuscular/ subcutaneous routes, respectively and were subsequently inoculated via oral route with the challenge strain at 3 wpi to investigate the protective efficacy of the Salmonella Gallinarum ghosts against virulent challenge. The birds were subsequently observed for mortality for 14 days post-challenge. Immunized groups $\mathrm{B}, \mathrm{C}$, and D showed significant protection against the challenge compared to the non-immunized group A (Table 4). The immunized groups exhibited significantly lower mortality than the non-immunized control group A, which showed $73 \%$ mortality (Table 4). The percent mortality rates for groups $\mathrm{B}, \mathrm{C}$, and $\mathrm{D}$ were $14.0 \%, 6.6 \%$, and $6.6 \%$, respectively. At day 14 post-challenge, in each group the surviving chickens were euthanized for postmortem examination to evaluate the protective efficacy of the ghost vaccine. The presence of hepatic and splenic enlargement with white necrotic spots was scored from 0 to 3 as described in "Materials and Methods". The mean organ lesion scores of the immunized groups were compared to those of the non-immunized control group $\mathrm{A}$. All the immunized groups (B, C, and D) had significantly lower lesion scores than those of the control group $\mathrm{A}$ (Table 4). The challenge strain was not isolated from the internal organs of any of the chicks of groups at day 14 post challenge.

\section{Discussion}

To vaccinate against FT, it is necessary that the vaccine contains either live Salmonella vaccine strains of reduced virulence, or inactivated organisms. In the present study we constructed a Salmonella Gallinarum ghost vaccine and evaluated its protective efficacy in chickens. Bacterial ghost (BG) represents a relatively novel concept for genetically engineered vaccine technology. However, this new ghost system has been rarely used in poultry. BG are devoid of all cytoplasmic content but preserve their natural outer membranes with highly immunogenic lipopolysaccharide structures. As reported 


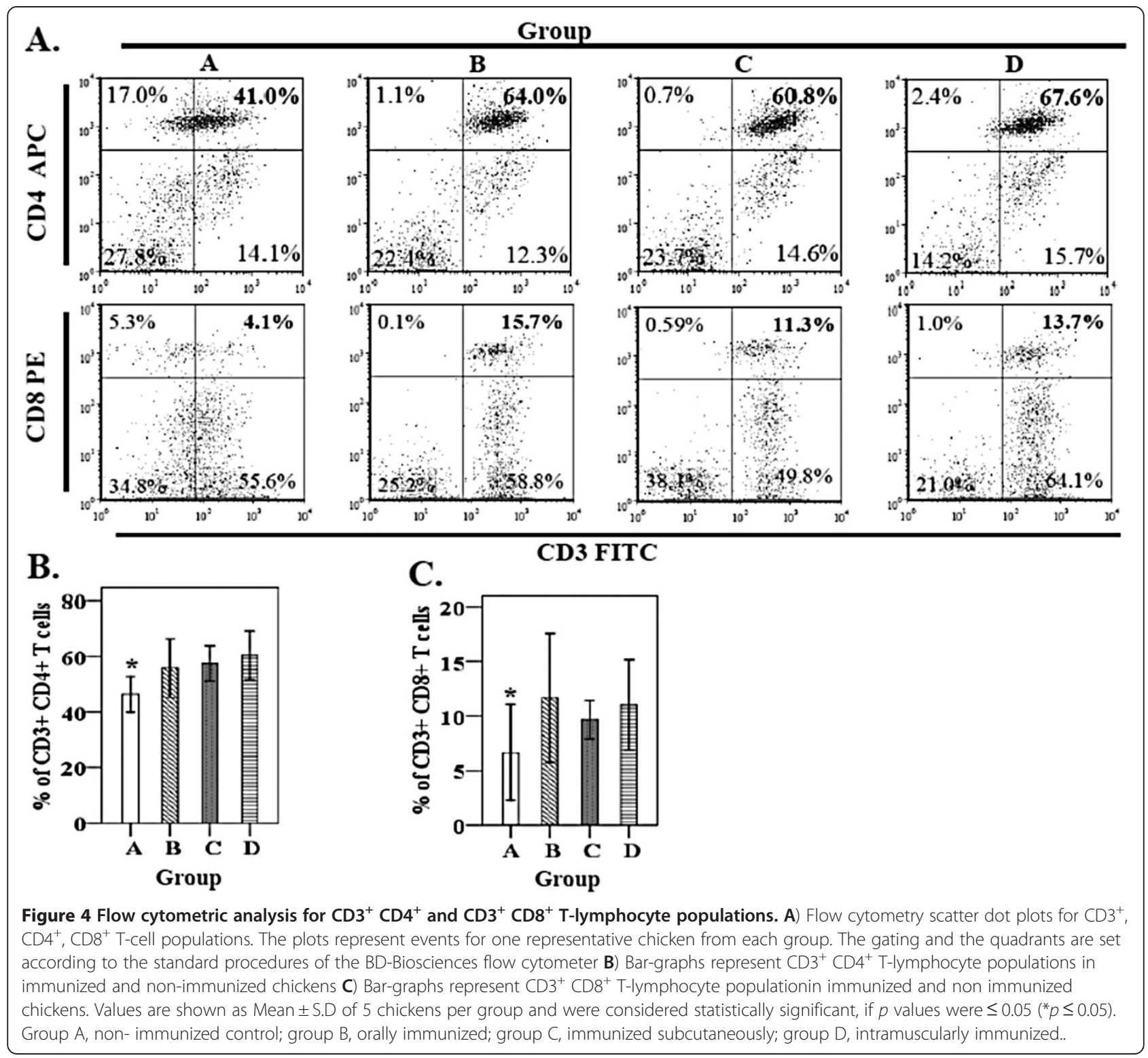

in this paper, we succeeded in producing Salmonella Gallinarum ghosts as a result of lysis gene $E$ expression followed by formation of transmembrane tunnel structures through which the cytoplasmic contents were expelled, leaving behind the empty cell envelopes (Figure 1). Culture solutions of Salmonella Gallinarum ghosts produced by this method did not contain any viable cells $24 \mathrm{~h}$ after lysis induction and no morphological changes were observed by SEM or TEM. The onset of lysis was observed $6 \mathrm{~h}$ after the temperature was increased to $42{ }^{\circ} \mathrm{C}$ with decreases in both $\mathrm{OD}_{600}$ and cell viability. Generally, strains carrying a conventional ghost cassette system are able to survive at $28{ }^{\circ} \mathrm{C}$ $[37,38]$ whereas the ghost strain used in the present study was grown at $37{ }^{\circ} \mathrm{C}$, which is the optimum temperature for normal growth of Salmonella [30], since sub-optimal growth temperatures reduce the rate of division and thus slowdown the production process of bacterial ghosts. Previous studies have indicated that growth of Salmonella at lower than $30{ }^{\circ} \mathrm{C}$ affected the lipopolysaccharide O-antigen size and distribution [39] and reduced the expression of certain fimbriae [40]. These changes in the surface structures may account for the alteration in antigenicity of Salmonella Gallinarum ghost. A ghost strain, which was initially grown at $37{ }^{\circ} \mathrm{C}$ and then exposed to $42{ }^{\circ} \mathrm{C}$ to promote the lysis, resulted in efficient induction of immune responses [16]. Based on this, the present study assumed that the Salmonella Gallinarum cells when grown under normal optimal temperatures required for Salmonella growth before the 
Table 3 Safety dose evaluation of the Salmonella Gallinarum ghost vaccine candidate in chickens

\begin{tabular}{llc}
\hline Group & Dose $^{\mathbf{a}}$ & Mortality after Challenge $^{\mathbf{b}}(\%)$ \\
\hline Vaccinated & $10^{7}$ & $7 / 15(46.6)$ \\
& $10^{8}$ & $5 / 15(33.5)$ \\
& $10^{9}$ & $4 / 15(26.6)$ \\
Control & - & $11 / 15(73.6)$ \\
\hline
\end{tabular}

${ }^{a}$ The birds were inoculated with $1 \times 10^{7}, 1 \times 10^{8}$ and $1 \times 10^{9}$ of the Salmonella Gallinarum ghost vaccine.

${ }^{\mathrm{b}}$ Number of dead birds upon challenge.

actual lysis induction could maintain their natural antigenic structure efficiently to trigger the desired immune responses.

It has been previously demonstrated that immunization with BG vaccines offer efficient protection against highly virulent strains in animal models [16,41]. Therefore, we further evaluated the safety and protective efficacy of the Salmonella Gallinarum ghosts as a vaccine candidate against infection with a wild-type strain in chickens immunized with Salmonella Gallinarum ghosts via either oral, intramuscular, or subcutaneous routes. None of the immunized groups showed any adverse reactions after vaccination which suggests that the ghost vaccine used in the present study could be safely administered in chickens without any detrimental effects. Immunization with the Salmonella Gallinarum ghosts offered significant protection against the virulent challenge based on mortality and the development of lesions (Table 4). Animals in all the immunized groups had significantly lower gross lesion scores compared to the non-immunized control group. Although no significant differences were observed among

Table 4 Mortality and gross lesion in the chickens post challenge

\begin{tabular}{|c|c|c|c|c|c|}
\hline \multirow[t]{4}{*}{ Group $^{a}$} & \multicolumn{5}{|c|}{ Challenge $^{\mathrm{b}}$} \\
\hline & \multicolumn{3}{|c|}{ Gross lesion $^{d}$} & \multicolumn{2}{|c|}{ Bacterial recovery } \\
\hline & \multirow{2}{*}{$\begin{array}{l}\text { Mortality } \\
\text { (\%) }\end{array}$} & \multirow{2}{*}{ Liver } & \multirow[t]{2}{*}{ Spleen } & Liver & Spleen \\
\hline & & & & \multicolumn{2}{|c|}{$\overline{\text { Positive No.Positive No }}$} \\
\hline $\bar{A}$ & $11 / 15(73)$ & $2.2 .16 \pm 1.46^{\mathrm{e}^{* *}}$ & $1.70 \pm 1.46^{*}$ & $0 / 5^{f}$ & $0 / 5$ \\
\hline B & 2/15 (14) & $1.06 \pm 0.96$ & $0.66 \pm 1.12$ & $0 / 5$ & $0 / 5$ \\
\hline C & $1 / 15(6.6)$ & ) $0.60 \pm 0.98$ & $0.53 \pm 0.83$ & $0 / 5$ & $0 / 5$ \\
\hline D & $1 / 15(6.6)$ & ) $0.53 \pm 0.89$ & $0.53 \pm 0.93$ & $0 / 5$ & $0 / 5$ \\
\hline
\end{tabular}

${ }^{a}$ Immunization was performed at day 7 of age with the Salmonella Gallinarum ghost strain and the groups were designated as group A (non vaccinated), group B (orally immunized), group C (immunized subcutaneously) and group D (immunized intramuscularly). Fifteen birds were allocated per group.

${ }^{b}$ Challenge was performed with a wild type Salmonella Gallinarum strain using $1 \times 10^{6}$ CFU after 21 days post vaccination.

${ }^{c}$ Number of dead birds upon challenge.

d Gross lesion was observed at day14 post challenge.

e Group lesion score (Mean \pm SD).

${ }^{f}$ Number of positive samples after enrichment culture. All values were considered to be significant if $p \leq 0.05$ or $0.01 .^{*} p<0.05,{ }^{* *} p<0.01$ vs. non vaccinated group $A$. the immunized groups, the mortality rates and gross lesion scores for all the immunized groups differ significantly compared to those of the non-immunized group. The present study results are promising and may indicate that the newly constructed Salmonella Gallinarum ghost vaccine could be an effective measure to prevent FT. However, the advantages of the presently constructed Salmonella Gallinarum ghost vaccine over the previously reported live [10] or subunit vaccines [6] should be validated. This could be achieved via comparative evaluation studies between the Salmonella Gallinarum ghost vaccine and other live/inactivated vaccines.

Salmonella Gallinarum antigen-specific humoral and cellular immune responses induced by vaccination have been correlated with the protective efficacy of live vaccines against FT in chickens $[33,42]$. Unlike other inactivated vaccines, $B G$ retain all relevant antigens found on live bacteria which can be recognized and engulfed by dendritic cells and macrophages in immunized animals, thereby stimulating humoral and cell-mediated immune responses [41]. Therefore, our study further investigated the humoral and cellular immune responses in chickens after immunization with the Salmonella Gallinarum ghosts. Our data indicate that immunization with the Salmonella Gallinarum ghosts efficiently induced systemic IgG responses in all immunized groups whereas mucosal sIgA levels were significantly elevated in group $\mathrm{B}$ (Figure 2a and b). The Salmonella Gallinarum ghost vaccine induced significantly high plasma IgG levels in chickens and the antibody levels continually increased until $3 \mathrm{wpi}$, indicating a strong systemic immune response in the chickens after immunization. Mucosal sIgA levels were significantly elevated in the orally immunized group. The stronger sIgA response was likely to be related to the fact that the oral route of immunization induces significantly higher mucosal secretory IgA levels compared to the other routes [43]. Thus, our data suggest that the oral immunization with the Salmonella Gallinarum ghosts had an additional advantage of inducing sIgA levels which serve as a first line of defense against enteric pathogens [43] and might play an important role in early elimination of Salmonella Gallinarum infections. Moreover, the role of sIgA or mucosal immunity in Salmonella Gallinarum clearance and systemic FT control has not been fully elucidated [42]. The present experimental data showing $\operatorname{sIgA}$ induction following oral immunization with $10^{10}$ Salmonella Gallinarum ghosts may offer useful information about the induction of intestinal SIgA antibodies although its role in protection against the Salmonella Gallinarum clearance in the chicken is a matter of interest. Presently, our study indicates that immunization with an inactivated Salmonella Gallinarum ghost vaccine via oral route is capable of inducing mucosal immunity. 
Cell-mediated immune responses play a central role in protecting against intracellular bacterial pathogens $[16,33,44]$. Salmonella Gallinarum is an intracellular pathogen, and has been reported to invade the mononuclear phagocyte system and survive in chicken macrophages [30]. Therefore, immunization against Salmonella Gallinarum infection requires a vaccine that can efficiently prevent the disease by inducing immune responses at the cellular level. The correlation between cellular immunity and high survival rates in chickens after a virulent Salmonella Gallinarum challenge has been reported, and measurements of cell-mediated immune responses is an important index of protection $[30,42]$. To evaluate the cellular immune responses, the present study performed an antigen-specific lymphocyte activation assay and, $\mathrm{CD}^{+}{ }^{+} \mathrm{CD} 4^{+}$and $\mathrm{CD}^{+}{ }^{+} \mathrm{CD} 8^{+} \mathrm{T}$ cell populations among the PBMC of the immunized and non-immunized groups. Our data showed that all immunized groups showed significant lymphocyte activation responses upon antigen stimulation compared to the non-immunized groups. These lymphocyte activation responses clearly suggest that the lymphocytes (either B or $\mathrm{T}$ cells) from the immunized birds were activated upon antigen stimulation, possibly due to circulating memory cells in the peripheral blood of the immunized birds. These findings may indicate that the Salmonella Gallinarum ghost vaccine could be as efficient as the other vaccines for activation of the lymphocytes.

In addition, we investigated dynamic changes in $\mathrm{CD} 4^{+}$ and $\mathrm{CD}^{+}{ }^{+}$-lymphocyte populations in the immunized and non-immunized chickens. Analysis of the T cell populations in the respective vaccination groups revealed significant changes in $\mathrm{T}$ cell population profiles in all vaccinated groups compared to the nonimmunized control group A (Figure 4). Our data show that vaccination with Salmonella Gallinarum ghosts induced a relative increase in the percentages of CD3 ${ }^{+} \mathrm{CD}_{4}{ }^{+}$and $\mathrm{CD}^{+} \mathrm{CD} 8+\mathrm{T}$ lymphocytes in the chickens. $\mathrm{CD}^{+} \mathrm{T}$ cells represent a major $\mathrm{T}$ cell population and are well known as $\mathrm{T}$ helper cells mainly associated with the Th1 and Th2 immune responses via the production of Th1 cytokines and increased antibody secretion, respectively [45]. CD8+ T cells, also known as cytotoxic T cells, play an important role in immune protection against intracellular bacterial parasites such as Listeria monocytogenes, Mycobacterium tuberculosis, and Salmonella enterica [45-47]. Thus, enhanced populations of these T cells in the immunized host are involved in protecting against intracellular pathogens. Increased $\mathrm{CD}^{+}$and $\mathrm{CD}^{+} \mathrm{T}$ cell populations in the chickens immunized with a live Salmonella Gallinarum mutant have been shown to correlate with protection against FT [36]. The data presented in this paper demonstrate that all immunized groups had $\mathrm{CD}_{4}^{+}$and $\mathrm{CD} 8^{+} \mathrm{T}$ cell populations and the percentages were significantly higher than those of the control group (Figure 4), indicating a strong cellmediated immune response. The immune response observed in our study was similar to that induced in BG-vaccinated animals [14,37], and involved the generation of $\mathrm{CD}^{+}$and $\mathrm{CD}^{+} \mathrm{T}$ cells. Taken together, our data demonstrate that vaccination with the Salmonella Gallinarum ghosts induced a significant cellular immune response characterized by effective antigen-specific lymphocyte activation with enhanced production of $\mathrm{T}$ helper $\left(\mathrm{CD}^{+}\right)$and cytotoxic $\left(\mathrm{CD}^{+}\right) \mathrm{T}$ lymphocytes. However, it would be interesting to know whether there was an increase in the actual lymphocyte populations in the immunized groups in response to immunization and the relative increase in the percentages of the $\mathrm{CD} 4^{+}$and $\mathrm{CD}^{+} \mathrm{T}$ cells should be recalculated back to the real numbers to know if the changes were due to increases in these lymphocyte subpopulations.

In conclusion, the present study demonstrates that the vaccination of chickens with the Salmonella Gallinarum ghosts is a safe approach for preventing FT without inducing adverse clinical symptoms. Immunization with the Salmonella Gallinarum ghost not only led to significant immune responses but also provided efficient protection against experimentally-induced systemic FT. Taken together, these findings suggest that the newly constructed Salmonella Gallinarum ghost appears to be a promising vaccine candidate and can be used as a safe and highly immunogenic vaccine against FT although further safety and efficacy trials on the field are needed.

\section{Competing interests}

The authors declare that they have no competing interests.

\section{Acknowledgements}

This work was supported by Mid-career Researcher Program through National Research Foundation (NRF) grant funded by the Ministry of Education, Science and Technology (MEST) (No. 2012-R1A2A4A01002318), Republic of Korea.

\section{Authors' contributions}

Conception and design of experiments: AC and JL. Conduction of experiments: AC, CJ and SK. Ghost cassette construction: SK. Animal experimentation and sample collection: $A C$ and CJ. Manuscript draft preparation and data analysis: AC, JL and SK. Study supervision: JL. All authors have read and approved the final manuscript.

Received: 4 July 2011 Accepted: 11 May 2012

Published: 23 May 2012

\section{References}

1. Barrow PA, Wallis TS: Vaccination of food animals. In Salmonella in domestic animals. Edited by Wray C, Wray A. Wallingford: CABl; 2000:323339.

2. Pomeroy BS, Nagaraja KV: Fowl Typhoid. In Diseases of Poultry. Edited by Calnek BW, Barnes HJ, Beard CW, Reid WM, Yoder HW Jr. Ames: lowa State University Press; 1991:87-99.

3. Basnet HB, Kwon HJ, Cho SH, Kim SJ, Yoo HS, Park YH, Yoon SI, Shin NS, Youn HJ: Reproduction of fowl typhoid by respiratory challenge with Salmonella Gallinarum. Avian Dis 2008, 52:156-159.

4. Wigley P, Hulme S, Powers C, Beal R, Smith A, Barrow P: Oral infection with the Salmonella enterica serovar Gallinarum $9 \mathrm{R}$ attenuated live vaccine as 
a model to characterize immunity to fowl typhoid in the chicken. BMC Vet Res 2005, 1:2

5. Zhang-Barber L, Turner AK, Dougan G, Barrow PA: Protection of chickens against experimental fowl typhoid using a nuoG mutant of Salmonella serotype Gallinarum. Vaccine 1998, 16:899-903.

6. Bouzoubaa K, Nagaraja KV, Kabbaj FZ, Newman JA, Pomeroy BS: Feasibility of using proteins from Salmonella gallinarum vs. $9 R$ live vaccine for the prevention of fowl typhoid in chickens. Avian Dis 1989, 33:385-391.

7. Rosu V, Chadfield MS, Santona A, Christensen JP, Thomsen LE, Rubino S, Olsen JE: Effects of crp deletion in Salmonella enterica serotype Gallinarum. Acta Vet Scand 2007, 49:14.

8. Shah DH, Shringi S, Desai AR, Heo EJ, Park JH, Chae JS: Effect of metC mutation on Salmonella Gallinarum virulence and invasiveness in 1-dayold White Leghorn chickens. Vet Microbiol 2007, 119:352-357.

9. Smith HW: The use of live vaccines in experimental Salmonella Gallinarum infection in chickens with observation on their interference effect. J Hyg 1956, 54:419-432.

10. Silva EN, Snoeyenbos GH, Weinack OM, Smyser CF: Studies on the use of 9R strain of Salmonella Gallinarum as a vaccine in chickens. Avian Dis 1981, 25:38-52.

11. Barrow PA, Huggins MB, Lovell MA: Host specificity of Salmonella infection in chickens and mice is expressed in vivo primarily at the level of the reticuloendothelial system. Infect Immun 1994, 62:4602-4610.

12. Jeong JH, Song M, Park SI, Cho KO, Rhee JH, Choy HE: Salmonella enterica serovar Gallinarum requires $p p G p p$ for internalization and survival in animal cells. J Bacteriol 2008, 190:6340-6350.

13. Ferguson M, Wood DJ, Minor PD: Antigenic structure of poliovirus in inactivated vaccines. J Gen Virol 1993, 74:685-690.

14. Melamed D, Leitner G, Heller ED: A vaccine against avian colibacillosis based on ultrasonic inactivation of Escherichia coli. Avian Dis 1991, 35:1722.

15. Kwon SR, Nam YK, Kim SK, Kim KH: Protection of tilapia (Oreochromis mosambicus) from Edwardsiellosis by vaccination with Edwardsiella tarda ghosts. Fish Shellfish Immunol 2006, 20:621-626.

16. Peng W, Si W, Yin L, Liu H, Yu S, Liu S, Wang C, Chang Y, Zhang Z, Hu S, Du $Y$ : Salmonella enteritidis ghost vaccine induces effective protection against lethal challenge in specific-pathogen-free chicks. Immunobiology 2011, 216:558-565

17. Lubitz W, Witte A, Eko FO, Kamal M, Jechlinger W, Brand E, Marchart J, Haidinger W, Huter V, Felnerova D, Stralis-Alves N, Lechleitner S, Melzer H, Szostak MP, Resch S, Mader H, Kuen B, Mayr B, Mayrhofer P, Geretschläger R, Haslberger A, Hensel A: Extended recombinant bacterial ghost system. J Biotechnol 1999, 73:261-273.

18. Szostak MP, Hensel A, Eko FO, Klein R, Auer T, Mader H, Haslberger A, Bunka S, Wanner G, Lubitz W: Bacterial ghosts: nonliving candidate vaccines. J Biotechnol 1996, 44:161-170

19. Witte A, Wanner G, Bläsi U, Halfmann G, Szostak M, Lubitz W: Endogenous transmembrane tunnel formation mediated by phiX174 lysis protein $\mathrm{E}$. J Bacteriol 1990, 172:4109-4114.

20. Eko FO, Witte A, Huter $V$, Kuen B, Fürst-Ladani S, Haslberger A, Katinger A, Hensel A, Szostak MP, Resch S, Mader H, Raza P, Brand E, Marchart J, Jechlinger W, Haidinger W, Lubitz W: Newstrategies for combination vaccines based on the extended recombinant bacterial ghost system. Vaccine 1999, 17:1643-1649.

21. Eko FO, Mayr UB, Attridge SR, Lubitz W: Characterization and immunogenicity of Vibrio cholerae ghosts expressing toxin-coregulated pili. J Biotechnol 2000, 83:115-123.

22. Jalava K, Hensel A, Szostak M, Resch S, Lubitz W: Bacterial ghosts as vaccine candidates for veterinary applications. J Control Release 2002, $85: 17-25$.

23. Jalava K, Eko FO, Riedmann E, Lubitz W: Bacterial ghosts as carrier and targeting systems for mucosal antigen delivery. Expert Rev Vaccines 2003 2:45-51.

24. Mayr UB, Haller C, Haidinger W, Atrasheuskaya A, Bukin E, Lubitz W, Ignatyev G: Bacterial ghosts as an oral vaccine: a single dose of Escherichia coli 0157:H7 bacterial ghosts protects mice against lethal challenge. Infect Immun 2005, 73:4810-4817.

25. Witte A, Wanner G, Sulzner M, Lubitz W: Dynamics of PhiX174 protein Emediated lysis of E. coli. Arch Microbiol 1992, 157:381-388.

26. Ra CH, Kim YJ, Park SJ, Jeong CW, Nam YK, Kim KH, Kim SK: Evaluation of optimal culture conditions for recombinant ghost bacteria vaccine production with the antigen of Streptococcus iniae GAPDH. J Microbiol Biotechnol 2009, 19:982-986.

27. Hayat MA: Introduction to biological scanning electron microscopy. Baltimore: University Park Press; 1978:242-243.

28. Matsuguchi M, Takeya K, Umeda A, Amako K, Watabe T, Aita S: Optimal condition for observing bacterial flagella by the scanning electron microscope. J Electron Microsc 1977, 26:343-344.

29. Johnson J, Reid WM: Anticoccidial drugs: lesion scoring techniques in battery and floor-pen experiments with chickens. Exp Parasitol 1970, 28:30-36.

30. Matsuda K, Chaudhari AA, Kim SW, Lee KM, Lee JH: Physiology, pathogenicity and immunogenicity of lon and/or cpxR deleted mutants of Salmonella Gallinarum as vaccine candidates for fowl typhoid. Vet Res 2010, 41:59.

31. Song $H$, Yan $R, X u L$, Song X, Shah MA, Zhu H, Li X: Efficacy of DNA vaccines carrying Eimeria acervulina lactate dehydrogenase antigen gene against coccidiosis. Exp Parasitol 2010, 126:224-231.

32. Porter RE Jr, Holt PS: Use of a pilocarpine-based lavage procedure to study secretory immunoglobulin concentration in the alimentary tract of White Leghorn chickens. Avian Dis 1992, 36:529-536.

33. Rana N, Kulshreshtha RC: Cell-mediated and humoral immune responses to a virulent plasmid-cured mutant strain of Salmonella enterica serotype Gallinarum in broiler chickens. Vet Microbiol 2006, 115:156-162.

34. Gogal RM Jr, Ahmed SA, Larsen CT: Analysis of avian lymphocyte proliferation by a new, simple, nonradioactive assay (lympho-pro). Avian Dis 1997, 41:714-725.

35. Crouch SP, Kozlowski R, Slater KJ, Fletcher J: The use of ATP bioluminescence as a measure of cell proliferation and cytotoxicity. J Immunol Methods 1993, 160:81-88.

36. Park $\mathrm{SI}$, Jeong $\mathrm{JH}$, Choy HE, Rhee $\mathrm{JH}, \mathrm{Na}$ HS, Lee TH, Her M, Cho KO, Hong $Y$ : Immune response induced by ppGpp-defective Salmonella enterica serovar Gallinarum in chickens. J Microbio/ 2010, 48:674-681.

37. Jechlinger W, Haller C, Resch S, Hofmann A, Szostak MP, Lubitz W: Comparative immunogenicity of the hepatitis B virus core 149 antigen displayed on the inner and outer membrane of bacterial ghosts. Vaccine 2005, 23:3609-3617.

38. Tu FP, Chu WH, Zhuang XY, Lu CP: Effect of oral immunization with Aeromonas hydrophila ghosts on protection against experimental fish infection. Lett Appl Microbiol 2010, 50:13-17.

39. McConnell M, Wright A: Variation in the structure and bacteriophageinactivating capacity of Salmonella Anatum lipopolysaccharide as a function of growth temperature. J Bacterio/ 1979, 137:746-751.

40. Thorns CJ, Sojka MG, Mclaren IM, Dibb-Fuller M: Characterisation of monoclonal antibodies against a fimbrial structure of Salmonella enteritidis and certain other serogroup $D$ salmonellae and their application as serotyping reagents. Res Vet Sci 1992, 53:300-308.

41. Wang X, Lu C: Mice orally vaccinated with Edwardsiella tarda ghosts are significantly protected against infection. Vaccine 2009, 27:1571-1578.

42. Matsuda K, Chaudhari AA, Lee JH: Evaluation of safety and protection efficacy on cpxR and lon deleted mutant of Salmonella Gallinarum as a live vaccine candidate for fowl typhoid. Vaccine 2011, 29:668-674.

43. Pasetti MF, Simon JK, Sztein MB, Levine MM: Immunology of gut mucosal vaccines. Immunol Rev 2011, 239:125-148.

44. Davison TF: Cell-mediated immunity: effector functions. In Poultry Immunology. Edited by Davison TF, Morris TR. London: Carfax Publishing Company; 1996:115-134.

45. Mittrucker HW, Kaufmann SH: Immune response to infection with Salmonella typhimurium in mice. J Leukoc Biol 2000, 67:457-463.

46. Flynn JL, Goldstein MM, Triebold KJ, Koller B, Bloom BR: Major histocompatibility complex class I-restricted T cells are required for resistance to Mycobacterium tuberculosis infection. Proc Natl Acad Sci 1992, 89:12013-12017.

47. Roberts AD, Ordway DJ, Orme IM: Listeria monocytogenes infection in beta 2 microglobulin-deficient mice. Infect Immun 1993, 61:1113-1116.

\section{doi:10.1186/1297-9716-43-44}

Cite this article as: Chaudhari et al:: Construction of a Salmonella Gallinarum ghost as a novel inactivated vaccine candidate and its protective efficacy against fowl typhoid in chickens. Veterinary Research 2012 43:44 